\title{
EL ECOTURISMO: QUIMERA O REALIDAD DE DESARROLLO en la Reserva de la Biosfera Ría Celestún, México
}

\section{Ecotourism: Chimera or Reality of Development in the Biosphere Reserve Ria Celestun, Mexico}

\author{
Manuel Jesús Pinkus-Rendón \\ Miguel Ángel Pinkus-Rendón
}

\begin{abstract}
Resumen: El ecoturismo se define como una de las alternativas dentro del desarrollo sustentable que puede incidir positivamente tanto en la conservación de los recursos naturales, como en la economía de las comunidades. En el presente trabajo se reflexiona sobre el impacto del ecoturismo en las comunidades ubicadas en la Reserva de la Biosfera Ría Celestún, en la Península de Yucatán. Para llegar a esta meta, se llevaron a cabo entrevistas semiestructuradas a los prestadores de servicios, referentes al uso ecoturístico de la reserva. Se observaron diferencias en la percepción de la conservación de los recursos naturales dependiendo de la capacitación recibida. Asimismo, se advirtió que existía una falta de coordinación entre las distintas autoridades, lo que influía negativamente en el manejo sustentable de la reserva y en las cooperativas turísticas.
\end{abstract}

Palabras clave: turismo alternativo, poblaciones, pescadores, áreas naturales protegidas.

Abstract:Ecotourism is defined as one of the alternatives of sustainable development that can have apositive impactonboth, the conservation of natural resources and the economy of communities. The aim of this work is to reflect on the impact of ecotourism on communities within the Biosphere Reserve Ria Celestun in the Peninsula of Yucatan, Mexico. To reach this goal, semistructured interviews with service providers were conducted, regarding ecotourism use within the reserve. The results show significant differences in the perception of natural resources of services providers, which varied depending on the knowledge they have gained through training. A lack of coordination between the different levels of government authorities has influenced the way the reserve and tourist cooperatives are managed.

Keywords: alternative tourism, populations, fishermen, protected natural areas.

\footnotetext{
Manuel Jesús Pinkus Rendón, profesor-investigador en el Centro de Investigaciones Regionales Dr. Hideyo Noguchi de la Universidad Autónoma de Yucatán, México. Temas de especialización: desarrollo sustentable, turismo, impactos en áreas naturales protegidas y globalización. Correo electrónico: mpinkus@correo.uady.mx y yucpinkus@yahoo.com.mx.

Miguel Ángel Pinkus Rendón, investigador en el Centro Peninsular en Humanidades y Ciencias Sociales de la Universidad Nacional Autónoma de México. Temas de especialización: conocimiento local, cambio de uso del suelo y áreas naturales protegidas. Correo electrónico: mapinkus@humanidades.unam.mx y cdcaronte@yahoo.com.
}

Enviado a dictamen: 21 de enero de 2014. Aprobación: 08 de septiembre de 2014. Revisiones: 2. 


\section{Introducción}

E n el marco del discurso sobre desarrollo sustentable, un nuevo elemento se ha sumado a la expansión de la industria turística global: el ecoturismo. ${ }^{.}$Según la International Ecotourism Society (1990), ecoturismo significa "viajar en forma responsable a las áreas naturales, conservar el ambiente y mejorar el bienestar de la comunidad local. El ecoturismo está a favor de la conservación que conecta a las comunidades y el recorrido sostenible". Este tipo de turismo "resuelve las necesidades de los actuales turistas que llegan a las regiones [áreas naturales] mientras que protege y realza las oportunidades para el futuro [de los habitantes locales]" (The International Ecotourism Society, 1990).

Desde esta perspectiva, el ecoturismo o turismo ecológico, como también se le conoce, se vislumbró como la actividad capaz no sólo de coadyuvar a la generación de empleos e ingresos para las poblaciones que habitan en el seno de las reservas naturales, sino también a la promoción de la conservación y el aprovechamiento del entorno natural y cultural, lo que se tradujo en la proliferación de áreas naturales protegidas y en el fomento del ecoturismo como herramienta para lograr el desarrollo sustentable. Según Daltabuit (2000: 44) y la Comisión Nacional de Áreas Naturales Protegidas (CONANP), esta modalidad de turismo, a diferencia del turismo masivo, debe ser más sensible y provocar menos efectos negativos en el ambiente y en la cultura local, a la vez que debe contribuir a la conservación y al desarrollo sustentable de las áreas naturales protegidas y de áreas con otras modalidades de conservación, como una alternativa económica que beneficie a las comunidades y a los usuarios locales (CONANP, 2006: 6).

En este contexto, el Estado mexicano ha promovido ampliamente la actividad ecoturística en las últimas tres décadas como alternativa para contribuir al desarrollo económico del país. En este sentido, desde instancias gubernamentales se han definido líneas de acción para el desarrollo de proyectos de ecoturismo en áreas naturales protegidas a través de programas nacionales y estatales, que se deben emprender con un enfoque sustentable de aprovechamiento responsable, distributivo y rentable, que fortalezca el turismo como producto (CONANP, 2000: 16-18).

En México, la Secretaría de Turismo y la Comisión Nacional para el Desarrollo de los Pueblos Indígenas son las instituciones gubernamentales que promueven la actividad ecoturística como una forma de desarrollo para pueblos rurales y pequeños donde residen indígenas, que posean riqueza cultural, vestigios arqueológicos, playas, fauna, flora y paisajes, como una alternativa para lograr el crecimiento económico de dichos pueblos y así complementar sus ingresos, que tradicionalmente provienen, en especial, de actividades primarias como la pesca, la ganadería y la minería (INEGI, 2008: 10). No obstante, esta actividad puede verse de dos formas antagónicas: una negativa, que se traduce en la destrucción de frágiles ecosistemas, en pérdida de biodiversidad y en alteración, modificación o pérdida de valores sociales y culturales, además de contribuir a la inflación, cambiar estilos de vida locales o causar contaminación; y una positiva: la promoción de empleos e intercambios económicos para beneficio del país, como fuente de recursos para mantener reservas a través de la fabricación de artesanías destinadas al turismo, el trabajo y el desarrollo económico y social de las comunidades en donde se pretende llevar a cabo (Mújica, 2005), en particular en aquellas que se encuentran dentro de las áreas naturales protegidas, como el caso que se presenta en este trabajo: la Reserva de la Biosfera Ría Celestún.

Esta reserva fue declarada refugio faunístico en 1979, reserva especial de la biosfera en 1988 y reserva de la biosfera el 27 de noviembre de 2000 (CONANP, 2002: 6).

En este contexto, la CONANP publicó en 2002 el Programa de Manejo de la reserva con la finalidad de promover el desarrollo de actividades productivas alternativas a la pesca y a la producción de sal, para de esta manera elevar el nivel de vida de la población y al mismo tiempo favorecer un uso racional de los recursos de esta área natural (CONANP, 2002). Entre los objetivos del programa se encontraban los siguientes: a) recuperar aquellos ecosistemas que hayan sido disminuidos por la acción del hombre, b) desarrollar actividades turísticas que sean compatibles con los objetivos de manejo, y c) 
fomentar la participación activa de la población local en la conservación de los recursos naturales de la reserva.

El presente trabajo tiene como objetivo conocer, analizar y discutir si el ecoturismo puede ser la vía para valorar y proteger la diversidad natural y cultural, a través de la educación ambiental de los actores involucrados, o bien sólo se trata de una quimera de desarrollo sustentable.

\section{Área de estudio}

La Reserva de la Biosfera Ría Celestún se localiza en el extremo noroccidental de la Península de Yucatán, en México. Tiene una superficie de 81482.33 hectáreas y se localiza entre los municipios de Celestún y Maxcanú, en el estado de Yucatán, y Calkiní en el estado de Campeche (ver mapa 1). Su importancia biológica reside en la diversidad de ambientes que presenta: manglares, petenes, vegetación de duna costera, sabana y selva baja inundable. Dicha variedad ecosistémica sostiene una diversidad faunística representativa de la región. Destaca su avifauna, compuesta por 304 especies, residentes y migratorias, entre las que sobresalen las aves de costa y pantanos, como garzas (Ardeidae), patos (Anatidae), gaviotas (Laridae) y una riqueza de especies migratorias paserinas y playeras que provienen de los vecinos países del norte del continente, Estados Unidos y Canadá, en su ruta migratoria de invierno. Es importante remarcar que, en México, esta zona, al igual que la Reserva de la Biosfera Ría Lagartos, es un área para el descanso, la alimentación y la reproducción del flamenco rosado (Phoenicopterus ruber ruber) (CONANP, 2002: 3-4), el ave más representativa de la región, que tiene una gran importancia para el ecoturismo en la reserva dado que la mayoría de los visitantes viene con el objetivo de observarla.

Dentro de la reserva se localizan dos poblaciones: Celestún e Isla Arena, con 6810 y 754 habitantes respectivamente, según datos del XI Censo de población $y$ vivienda (INEGI, 2010). La actividad pesquera ocupa a la mayor parte de la población. El puerto de Celestún es el segundo en importancia en el estado de Yucatán por su volumen de captura de pesca y es uno de los principales productores de pulpo en el Golfo de México. En los últimos veinticinco años se ha desarrollado una actividad turística enfocada en la riqueza natural de la Ría Celestún, particularmente en la observación de las parvadas de flamencos, cuyo crecimiento y especialización va en ascenso. Hasta el año 2002, fecha de publicación del Programa de Manejo, de esta actividad dependían más de cien familias. En relación con el número de visitantes, en 1998 se estimó la visita de más de quince mil turistas, y en 1999 se estimó un aproximado de veinticinco mil (CONANP, 2002). En 2012, se consideró que acudieron entre cincuenta mil y cincuenta y cinco mil visitantes, en especial durante la Semana Santa y durante los meses de vacaciones de verano.

\section{Materiales y métodos}

En la Reserva de la Biosfera Ría Celestún la actividad ecoturística se encuentra circunscrita a grupos conformados en cooperativas, las cuales están integradas, en su mayoría, por varones de edades que oscilan entre los 18 y los 50 años. Cada cooperativa ecoturística está compuesta por diez individuos que tienen permiso de la reserva para dar el servicio dentro de la ría. En este sentido, en Celestún operan ocho cooperativas de lancheros; en Isla Arena, tres agrupaciones. En cada una de las comunidades seleccionadas, Celestún e Isla Arena, se llevaron a cabo entrevistas semiestructuradas a dos grupos focales, conformados cada uno por trece lancheros que laboraban en las cooperativas turísticas, un grupo en el embarcadero de SECTUR y el otro en la playa. En las entrevistas se les preguntó sobre el ecoturismo, la presencia de los manejadores de la reserva en los poblados, los cursos de capacitación a los cuales fueron invitados, así como los cuidados que proporcionaban a la reserva y el uso de los sitios ecoturísticos. La recopilación de los datos se realizó de octubre de 2012 a mayo de 2014. Se utilizaron los métodos de bola de nieve, observación directa y muestreo por saturación para identificar a los informantes clave (Goodman, 1961; Martín-Crespo y Salamanca, 2007). 


\section{Resultados y discusión}

Los resultados muestran que un primer grupo de entrevistados, el integrado por los lancheros situados en el embarcadero, señaló haber recibido cursos de capacitación para prestar servicios turísticos en el marco de la preservación del patrimonio natural que representa dicha reserva, y un segundo grupo, los lancheros que se localizaban en la playa, indicó que nunca había recibido capacitación o adiestramiento para la prestación de dicho servicio. La comparación entre ambos grupos de prestadores de servicios ecoturísticos permitió dilucidar que las personas que se dedican a esta actividad, cuando se capacitan en educación ambiental, tienen la tendencia a adquirir conciencia sobre la importancia de la conservación de los recursos naturales y, por ende, sobre la preservación del patrimonio natural, lo que favorece la sustentabilidad en la reserva.

En la Reserva de la Biosfera Ría Celestún se realizan actividades turísticas desde hace más de tres décadas, con recorridos en lanchas para observar las aves, principalmente los flamencos. Actualmente, muchos de los lancheros se dedican sólo a realizar paseos turísticos, mientras que otros complementan esta actividad con otras, como la pesca o la captura de especies marinas, o se dedican a oficios como la hostelería o la albañilería. En ambas poblaciones, Celestún e Isla Arena, hay restaurantes y hoteles, además de cabañas ecoturísticas que, si bien se promueven como instalaciones ecológicas, la mayoría no cumple con las características de lo que se denomina como construcción ecoamigable. Este tipo de construcción implica una infraestructura en la cual se utilizan materiales y procesos de bajo impacto ambiental, con un diseño arquitectónico acorde con la región y con materiales propios de la zona (SECTUR, 2006), como puede verse en la foto 1 , en la que se muestra una cabaña ecoturística de Isla Arena.

De acuerdo con la información contenida en el Programa de Manejo 2002 de la reserva, antes de establecer controles en cuanto al uso de los recursos naturales en esa área, los paseos en lancha se realizaban de manera desorganizada y sin considerar el daño al entorno ambiental. Por ejemplo, las lanchas se acercaban demasiado a la vegetación - compuesta en su mayoría por mangle - y a las aves, que eran perturbadas con el ruido. De igual manera, se talaban los mangles con el fin de utilizar la madera para la construcción de viviendas y para cocinar. En menor medida, se hacía el desmonte de la vegetación con objeto de utilizar esos espacios vacíos para construir hoteles, cabañas y otros edificios, principalmente para ofrecer servicios de alojamiento y alimentación a los turistas que acudían a la zona. Acciones como éstas representan un gran problema, no sólo en la Reserva de la Biosfera Ría Celestún, sino en todo el planeta.

Para contrarrestar lo anterior, a través del Programa de Manejo 2002, se establecieron acuerdos con los lancheros y se propusieron políticas públicas para proteger los recursos ambientales de la región. Sin embargo, en la actualidad, la actividad ecoturística continúa impactando en la reserva, ya que los mismos lancheros entrevistados mencionaron que todavía algunos prestadores de servicios se acercaban a las aves "si los turistas lo desean[aban] y lo pagan[aban]". Indicaron que, en ocasiones, los lancheros se aproximaban tanto a las aves, con el fin de brindar al turista una mejor vista, que les causaban perturbación, lo que se reflejaba en un aumento del estrés y en la disminución del tiempo efectivo de alimentación (CONANP, 2002: 20).

Asimismo, se pudo observar, en las diferentes entradas para el trabajo de campo, que tanto turistas como lancheros arrojaban desechos líquidos y sólidos en la ribera de la ría, así como en la playa y el mar. Había basura, envases, bolsas de plástico y papel, así como derrames de combustible de los motores de las lanchas, o bien de la gasolina con la que las lavaban, lo que contribuía a la alteración del hábitat de los flamencos, lo cual, por consiguiente, podía conducir a una disminución del número de esas aves que ahí anidan. Los lancheros del embarcadero señalaron que el grupo de los lancheros de la playa no respetaba los lineamientos establecidos en el Programa de Manejo ya que, cuando trasladaban a los visitantes en lancha para observar las aves y los manglares, no llevaban contenedores para desperdicios, o bien no cobraban el derecho ambiental ${ }^{2}$ que estipulaba la SEMARNAT, pues no significaba un 
ingreso personal. No obstante, el mismo discurso de incumplimiento del reglamento fue aludido por los lancheros de la playa hacia los del embarcadero. En cuanto a las acciones que realizaban estos prestadores de servicios en torno al cuidado del ambiente, las aprendieron a través de pláticas informales y de lo que habían escuchado de otras personas, pero de ninguna manera mediante una capacitación formal. La negativa de esos lancheros a entregar recibos y cobrar derechos ambientales a cada visitante originaba una afectación en la evaluación y control de las capacidades de carga de la reserva para el cuidado del ambiente. De igual modo, la poca atención que prestaban los funcionarios encargados de la vigilancia del uso responsable de los recursos dificultaba aún más el esfuerzo para atender esa necesidad.

Así también, se continuaba con la construcción de alojamientos que no seguían la reglamentación del Plan de Manejo. Por ejemplo, algunas de las cabañas consideradas ecoturísticas en Isla Arena no cumplían con los requisitos mencionados como turismo ecoamigable, es decir, no estaban construidas con material propio de la región y no estaban hechas de manera que ahorraran energía eléctrica, ni contaban con suficiente ventilación. En este sentido, los dueños de las cooperativas y los encargados de las cabañas denominadas ecológicas afirmaron que, al recibir el apoyo económico por parte del gobierno, edificaron esas construcciones como les pareció que deberían ser, con base en sus conocimientos personales y sin orientación o capacitación alguna por parte de las autoridades en materia ambiental. Después de cuatro años de funcionamiento, estaban reinvirtiendo en la remodelación y mantenimiento de dichas cabañas; sin embargo, los ingresos que habían obtenido por los servicios de hospedaje y alimentación que ofrecían no eran lo suficientemente elevados como para constituir una verdadera alternativa de subsistencia. Es decir, se pudo constatar mediante la investigación que la actividad turística no era la principal actividad económica que les proveía de recursos para sostener a sus familias. Esto se debía en gran medida a la falta de capacitación, tanto para la prestación de los servicios y su promoción, como para la administración de la infraestructura.

Por otra parte, aquellos turistas que pudieran estar dispuestos a visitar Isla Arena no obtenían suficiente información del lugar debido a que los prestadores de servicios no realizaban la suficiente divulgación turística en los diversos medios, ni promovían entre los visitantes la concienciación sobre el cuidado del ambiente. Por lo general, la conciencia ecológica ya existía en los visitantes que acudían atraídos por la tranquilidad del lugar $y$, fuera de algunas recomendaciones en las páginas de internet y en folletos que los gobiernos local y estatal imprimían relacionados con el cuidado y preservación del medioambiente, se debe señalar que dicha información no se difundía. ${ }^{3}$ Asimismo, las dependencias de gobierno, tanto estatal como municipal, tampoco promovían la importancia de construir un tipo de instalaciones turísticas en pro del cuidado del entorno, en el que se utilizaran materiales que no contravinieran las normativas del Programa de Manejo y que no contrastaran estéticamente con el entorno.

En Isla Arena existe una cooperativa llamada Wotoch Aayin — casa del cocodrilo en lengua maya-, dedicada a criar y mantener cocodrilos. En el momento del trabajo de campo, ésta era manejada y administrada por un grupo de personas que real y decididamente se habían preocupado por mantener el equilibrio ecológico. Se habían capacitado para cuidar y conservar los cocodrilos a través de la asistencia a cursos, talleres y diplomados que les permitieron adquirir compromisos y tomar decisiones en su empresa, las cuales afectaban de manera positiva en la preservación del patrimonio natural que les rodeaba. Asimismo, transmitían esta información a los visitantes y a los pobladores con los que tenían contacto, por lo que ejercían también una influencia positiva en otras personas (ver foto 2 ).

Lo mencionado anteriormente concuerda con la opinión de numerosos investigadores de las ciencias sociales — como Cater, Weaver y Daltabuit, por mencionar algunos-, quienes señalan que el ecoturismo, más que lograr los beneficios ambientales y sociales mencionados, ha generado algunos problemas, como 
los siguientes: una rápida degradación ambiental en ecosistemas frágiles - hasta ahora bien conservadoso la alteración física en las riberas y suelos causada por las lanchas y vehículos motorizados, caballos o bestias de carga, bicicletas y caminantes. A causa de ello, la reacción de los suelos a estas presiones y cargas queda evidenciada por una serie de cambios físicos, como la erosión de los litorales y la compactación de suelos. Otros efectos negativos documentados son la basura generada e, incluso, los incendios que se han producido, sobre todo en sitios muy concurridos. La transformación de las culturas más marginales y la perpetuación de las inequidades sociales también son evidentes (Daltabuit, 2000: 46) porque, como en el caso que nos ocupa, sólo los dueños de las embarcaciones, los cooperativistas o los hoteleros recibían ganancias, mas no así los lancheros o jornaleros.

Ha de entenderse que el ecoturismo, en teoría, debe promover actividades a nivel local que no sólo mejoren la calidad de vida de los habitantes de la zona, sino que también fortalezcan la preservación de los recursos naturales. Sin embargo, no se ha cuantificado el impacto económico que el ecoturismo ocasiona en la Reserva de la Biosfera Ría Celestún; es decir, si realmente las actividades realizadas en el marco del ecoturismo contribuyen a elevar el nivel de vida de los habitantes. Por otra parte, en el caso de que el nivel de vida se eleve, no se ha documentado si éste conlleva un grado de desarrollo más alto ${ }^{4}$ o, por el contrario, contribuye a una mayor degradación del ambiente y los ecosistemas.

En este punto, la capacitación y la educación para cuidar y preservar el entorno natural y sus recursos resultan imperativas, pues el desarrollo de esta región no debe verse sólo como la superación de necesidades básicas, como vivienda, salud, alimentación, seguridad y educación, sino como el alcance de un crecimiento económico sostenido y la conservación y protección del medioambiente, en congruencia con lo que se conoce como desarrollo sustentable. Para lograrlo, es necesario eliminar la pobreza que, en ocasiones, conduce a la sobreexplotación de los recursos naturales, los cuales son determinantes en las posibilidades productivas por su papel como insumos en el proceso económico
(Constantino, 2007: 57). Pero el término sustentabilidad podría toparse con una contradicción debido a que el uso de los recursos naturales renovables ya constituye en sí una degradación del patrimonio natural, pues, aunque por ejemplo áreas de manglar han sido reforestadas, esto no ocurre a la misma velocidad que su explotación (Martínez y Roca, 2001: 367).

Otra problemática tangencial la constituye el hecho de que durante mucho tiempo se han documentado conflictos entre los habitantes de Celestún e Isla Arena, que se originaron por pugnas relativas a los límites geográficos establecidos para realizar actividades de pesca en cada población. ${ }^{5}$ Como se pudo observar en ambas localidades, los lancheros han llevado estas diferencias al ámbito turístico, pues se niegan a ofrecer el servicio de traslado de una población a otra. Además, durante las entrevistas y en la observación directa, se percibió que constantemente culpan a la población vecina de no cuidar los recursos naturales y de que su interés se centra en transportar al mayor número de visitantes en los recorridos en lancha con el propósito de "ganar" clientes.

Entre los diversos grupos y cooperativas dedicados a la actividad ecoturística que se observaron —ocho en Celestún y tres en Isla Arena-, se encontraron diferencias en sus actitudes y opiniones. Al preguntar sobre el cuidado de los ecosistemas y el conocimiento sobre la importancia de preservar el equilibrio ecológico en la reserva, así como sobre su interés por recibir capacitación y educación ambiental, el 50\% de los entrevistados consideraba que eran puntos de suma importancia; sin embargo, señalaron que no habían recibido capacitación por parte de ninguna dependencia del gobierno, o bien que ésta no les interesaba. Los pocos que sí habían recibido capacitación fueron los cooperativistas, quienes generalmente no tripulaban las lanchas, ya que preferían que otros se encargaran de los paseos mientras ellos iban de pesca en alta mar o bien se quedaban en sus casas.

Actualmente, la legislación ambiental establecida en el Programa de Manejo y en la Ley General del Equilibrio Ecológico y Protección al Ambiente pone énfasis en el desarrollo del ecoturismo y en el uso de 
los recursos naturales de una manera responsable, cuidadosa y respetuosa con el medioambiente, así como en la conservación de los ecosistemas de la región. Sin embargo, estos objetivos no pueden alcanzarse en la reserva sin la debida capacitación de los habitantes, no solamente de los prestadores de servicios - como ha sido el caso del 50\% de los 26 lancheros entrevistadossino de la comunidad en general que habita en la misma y donde seguirán viviendo sus descendientes. En el marco de las legislaciones vigentes, es imprescindible instruir a los habitantes de las poblaciones sobre el cuidado y uso de los recursos naturales y la preservación del ambiente y los ecosistemas.

\section{Conclusiones}

Si bien tenían una percepción de la importancia del cuidado del entorno, menos de la mitad de los lancheros, tanto en Celestún como en Isla Arena, procuraba en los hechos la preservación, limpieza y descontaminación, tanto de la ría como del mar y la playa. No contaban con los suficientes conocimientos formales para realizar y promover actividades que no dañaran los recursos naturales o para hacer un uso adecuado de ellos, ni conocían mecanismos para la disposición y el manejo de residuos. Señalaron que "no tiran basura porque no está bien", pero estrictamente hablando no tenían una idea clara de por qué no debían hacerlo y, en su caso, cuáles eran las consecuencias. Por ello, era difícil cambiar sus actitudes y convencerlos del peligro de depositar los residuos en las playas, el mar o el manglar. En este sentido, las estrategias para "fomentar la impartición periódica de cursos de capacitación en manejo y aprovechamiento sostenible y sustentable de recursos naturales, dirigidos a los habitantes del área y personal de la reserva" y "el desarrollo de talleres de participación comunitaria con el objetivo de difundir la importancia y objetivos de la reserva", que se señalan en el Programa de Manejo, resultan completamente apropiadas para este fin, pues las comunidades, los empleados y los funcionarios de la reserva y de las otras instancias que tienen relación directa con la Reserva de la Biosfera Ría Celestún necesitan instruirse para transmitir esos conocimientos, tanto en el seno familiar, como a los turistas que arriban a la reserva.

El proceso mediante el cual los habitantes de la Reserva de la Biosfera Ría Celestún pueden instruirse en el manejo, uso, cuidado y conservación de los recursos naturales debe ser continuo para que, de manera consciente, asuman la responsabilidad de la protección del medioambiente. Ya que es un hecho que la actividad ecoturística seguirá realizándose en esa región, es indispensable una adecuada educación ambiental entre los pobladores locales para la aprehensión de conocimientos y la adopción de conductas y actitudes que conduzcan a una mínima degradación y contaminación del medioambiente. Asimismo, la capacitación de los prestadores de servicios turísticos puede contribuir a que los visitantes comprendan la importancia de cuidar los recursos naturales de la reserva que visitan. En el caso de la cooperativa Wotoch Aayin, las actitudes de sus integrantes, relacionadas con la explotación de los recursos naturales, cambiaron conforme fueron adquiriendo nuevos conocimientos sobre el cuidado del medioambiente; los valores y los principios sobre ese cuidado se canalizaron para lograr que el ecoturismo fuera puesto en práctica de acuerdo con criterios de sustentabilidad. Asimismo, los encargados de dicha cooperativa empezaron a transmitir a otras personas dedicadas a la actividad ese entendimiento de los valores ecológicos, lo que resultaba en una mayor conciencia ambiental, según ellos mismos afirmaron.

Como se pudo comprobar, una mayor información eleva las posibilidades de lograr un mejor cuidado y una adecuada conservación de la biosfera cuando se desarrollan actividades ecoturísticas, a la vez que se mejora los niveles de vida de los pobladores. Se comparten los criterios de la SEMARNAT en cuanto a la competitividad de las empresas ecoturísticas, con los supuestos que conforma la sustentabilidad, tales como el equilibrio ecológico — cuidado y preservación del entorno-, el desarrollo socioeconómico — mejora del producto turístico, con el valor agregado de recomendaciones e instrucciones sobre el cuidado de los recursos naturales - y el mejoramiento de 
la sociedad - lo que comprende una disminución de los niveles de criminalidad y prostitución, la integración de los grupos sociales, la participación de la comunidad en la atención de los turistas y la preservación de la cultura, entre otros-. Con estas premisas, las poblaciones de estudio pueden obtener ventajas competitivas frente a aquellas poblaciones que carezcan de la adecuada capacitación ambiental, lo cual se traduce también en el mejoramiento de la imagen pública entre turistas nacionales e internacionales (CONANP, 2002). De igual forma, la educación ambiental puede ser la base para abatir el rezago social identificado en ambas localidades, lo que resultará en mejoras en los ámbitos económico, social, ambiental y cultural, así como en un incremento de los ingresos reales que los prestadores de servicios turísticos y las empresas ecoturísticas perciben, sin dejar de lado el cuidado de los ecosistemas de la reserva.

\section{Notas}

${ }^{1}$ Término acuñado por Héctor Ceballos-Lascuráin (Mader, 2013).

${ }^{2}$ La cuota de ingreso a la ría es de 1300 pesos por lancha para seis personas. Dichos recursos ingresan directamente a la reserva y se utilizan en obras, proyectos o equipamiento para su conservación. Carabias (2010: 35) menciona que buena parte de estos ingresos se utilizan también en señalización, capacitación de guías, programas de educación ambiental, folletos informativos, etcétera.

${ }^{3}$ De hecho, la Constitución política de los Estados Unidos Mexicanos establece en su artículo cuarto que "Toda persona tiene derecho a un medio ambiente sano para su desarrollo y bienestar. El estado garantizará el respeto a este derecho. El daño y deterioro ambiental generará responsabilidad para quien lo provoque en términos de lo dispuesto por la ley", pero las sanciones por contaminar no están muy claras y cuando existen no se aplican (Carbonell, 2007).

${ }^{4}$ El término desarrollo, en una acepción socioeconómica ambiental, Escobar (1997) lo define como "la condición social dentro de un país, en la cual las necesidades auténticas de su población se satisfacen con el uso racional y sostenible de recursos y sistemas naturales".

${ }^{5}$ Los artículos que dan cuenta de los pleitos y diferencias entre los pobladores de Celestún e Isla Arena están disponibles en http:/historico.eluniversal.com.mx/ search/index.php?q=agua\&seccion=Estados\&autor= JAVIER+CASTILLO \%2FCorresponsal;http://www . proceso.com.mx/?p=255017 y http://www.campeche. com.mx/noticias/campeche_noticias/sigue-pleitopor-pepinos-de-mar-en-costas-campechanas/21779 [consultadas el 25 de enero de 2013].

\section{Referencias bibliográficas}

Carabias, Julia, José Sarukhán, Javier de la Maza y Carlos Galindo (coords.) (2010), Patrimonio natural de México. Cien casos de éxito. México, CONABIO/SEMARNAT.

Carbonell, M. (2007), Constitución política de los Estados Unidos Mexicanos. Leyes y códigos de México. México, Porrúa.

CONANP (Comisión Nacional de Áreas Naturales Protegidas) (2002), Programa de manejo: Reserva de la Biosfera Ría Celestún. México, CONANP/SEMARNAT. CONANP (Comisión Nacional de Áreas Naturales Protegidas) (2006), Programa de turismo en áreas protegidas 2006-2012. México, CONANP/SEMARNAT. CONANP (Comisión Nacional de Áreas Naturales Protegidas) (2012), Región Península de Yucatán, "Ubicación de la RB Ría Celestún". México, CONANP. Disponible en http://pyucatan.conanp. gob.mx/mapceles.htm [consultado el 16 de abril de 2012].

Constantino, Roberto (2007), "Recursos naturales y sustentabilidad: una perspectiva institucional y de acción colectiva”. En Calva, José Luis (coord.), Sustentabilidad y desarrollo ambiental. México, Porrúa.

Daltabuit Godás, Magalí, Héctor Cisneros, Luz María Vázquez y Enrique Santillán (2000), Ecoturismo y desarrollo sustentable. Impacto en comunidades rurales de la selva maya. México, UNAM/CRIM.

Goodman, L. (1961), "Snowball sampling”. En The Annals of Mathematical Statistics, vol. 32, núm. 1, pp. 148-170. 
INEGI (Instituto Nacional de Estadística, Geografía e Información) (2010), Conteo de población. México, INEGI.

Mader, Ron (2013), "Campeón del ecoturismo. Una conversación con el Arq. Héctor Ceballos-Lascuráin”. En planeta.com. Disponible en http://www.planeta. com/ecotravel/weaving/hectorceballos2.html [consultado el ll de octubre de 2013].

Martín-Crespo Blanco, María Cristina y Ana Belén Salamanca Castro (2007), "El muestreo en la investigación cualitativa”. En Nure Investigación, núm. 27, marzo-abril.

Martínez Alier, Joan y Jordi Roca (2001), Economía, ecológica y política ambiental. México, FCE.

Mújica, Luís y Graciela Mesa (2005), "Estudio previo evaluación impacto ambiental del camino perilago
Chajari". En Ilustrados.com. Argentina, Asociación Ecologista río Macoreta. Disponible en http://www. ilustrados.com/publicaciones/EEkkyAkkZFJKUvdvoY. php\# [consultado el 2l de mayo de 2013].

SECTUR (Secretaría de Turismo) (2006), "¿Qué es el turismo de naturaleza?" En Turismo de naturaleza. México, Secretaría de Turismo. Disponible en http://www.sectur.gob.mx/work/ sites/sectur/resources/LocalContent/14011/4/ QueEsTurismoDeNaturaleza.pdf [consultado el 22 de septiembre de 2012].

The International Ecotourism Society (1990), What is Ecotourism? Estados Unidos, TIES. Disponible en http://www.ecotourism.org/what-is-ecotourism [consultado el 8 de noviembre de 2013]. 


\section{Foto 1. Cabaña ecoturística de Isla Arena}

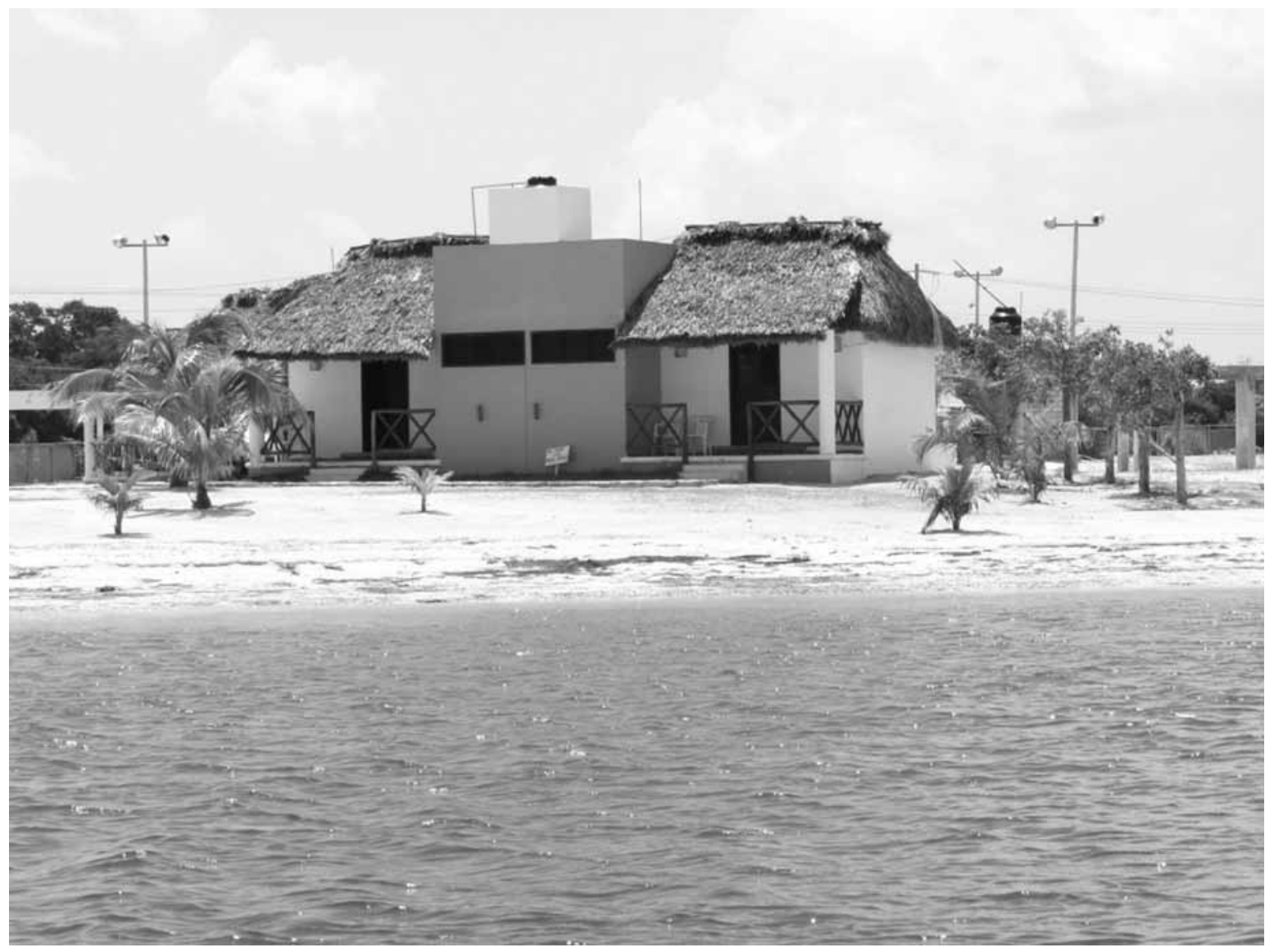

Fuente: los autores (2012). 


\section{Foto 2. Cría de cocodrilos en la cooperativa Wotoch Aayin, Isla Arena}

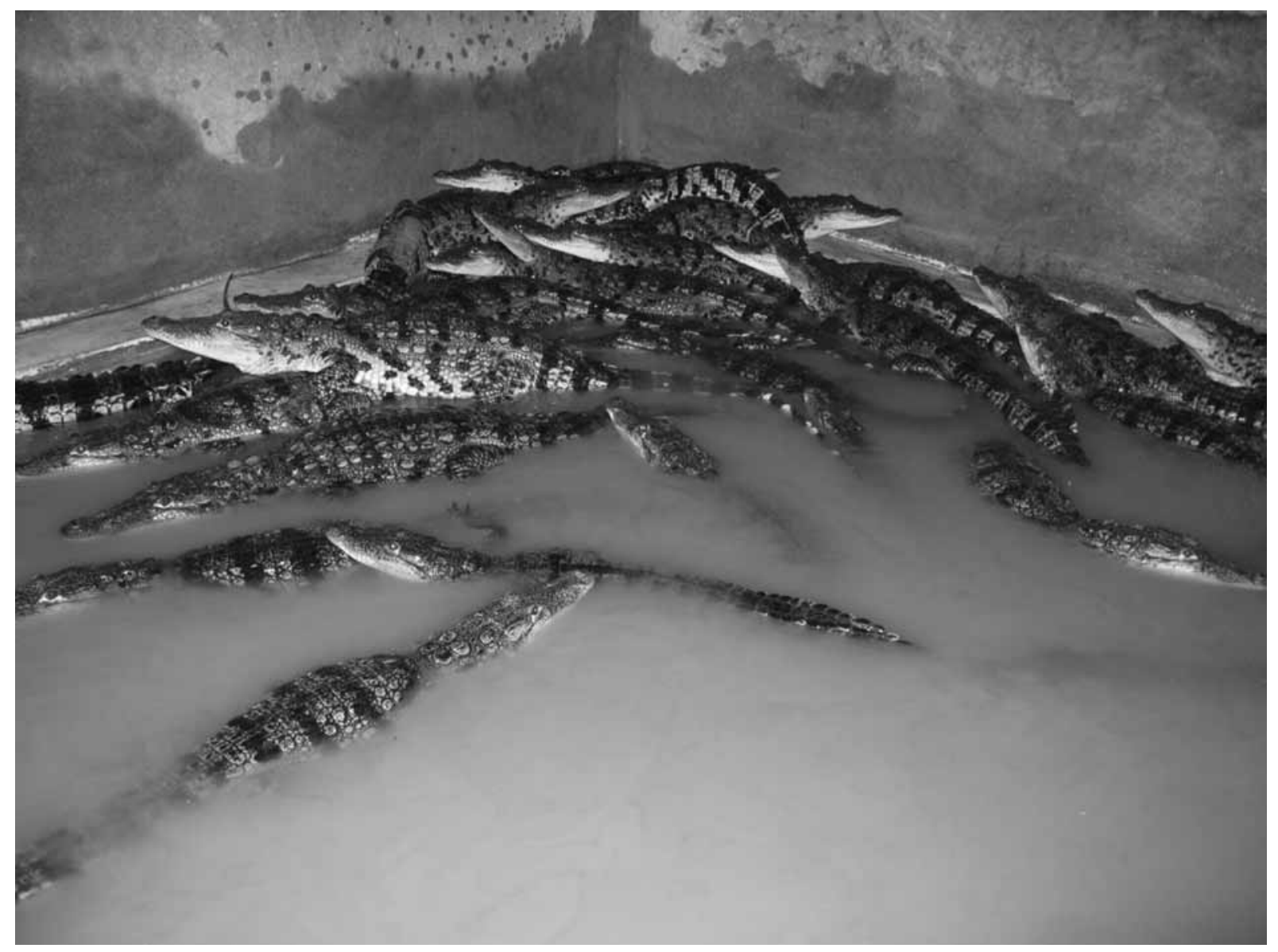

Fuente: los autores (2012). 
Mapa 1. Mapa de ubicación de la Reserva de la Biosfera Ría Celestún

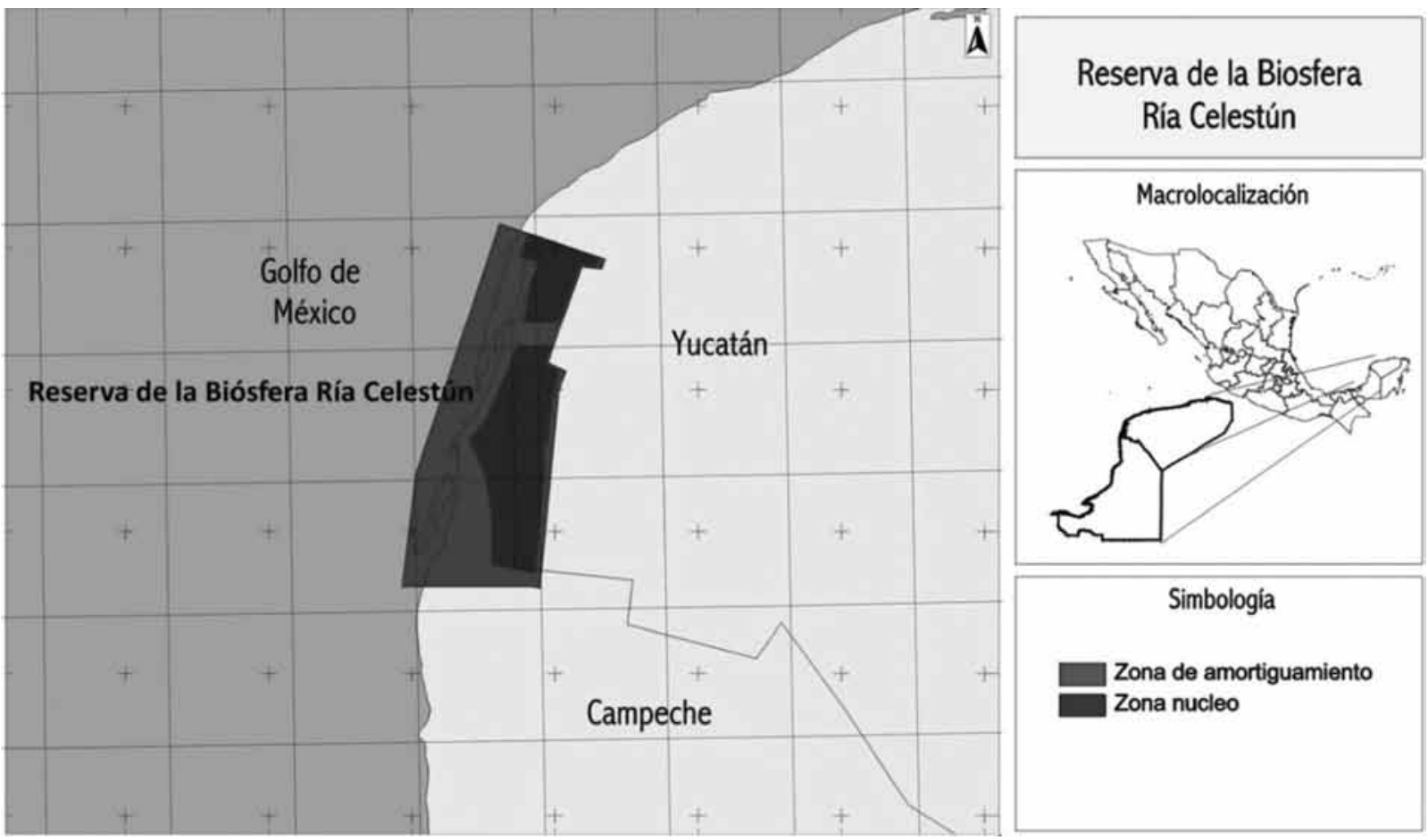

Fuente: CONANP-Yucatán (2012). 